\title{
The Hydrogen Evolution Reaction on Nanostructured Molybdenum Disulfide
}

\author{
C.A. Campos-Roldán ${ }^{1,2}$ and N. Alonso-Vante ${ }^{1 *}$ \\ ${ }^{1}$ IC2MP, UMR-CNRS 7285, University of Poitiers, 4 rue Michel Brunet, 86022 Poitiers, France. \\ ${ }^{2}$ Instituto Politécnico Nacional-ESIQIE, Laboratorio de Electroquímica y Corrosión, UPALM, 07738, CDMX, \\ México
}

*Corresponding author: N. Alonso-Vante, e-mail: nicolas.alonso.vante@univ-poitiers.fr

Received April $3^{\text {rd }}, 2018$; Accepted April 9 $9^{\text {th }}, 2019$.

DOI: http://dx.doi.org/10.29356/jmcs.v63i3.533

\begin{abstract}
This short review analyzes the catalytic activity of nanostructured molybdenum disulfide $\left(\mathrm{MoS}_{2}\right)$. The phase transition of nanostructured $\mathrm{MoS}_{2}$ semiconductor from $2 \mathrm{H}$ (hexagonal) to $1 \mathrm{~T}$ (trigonal) leads to a metal-like material through so-called surface defect engineering by chemical exfoliation. The 1T phase was found to be active for the electrocatalytic hydrogen evolution reaction (HER). Various key aspects vis-à-vis the $\mathrm{MoS}_{2}$ synthesis, characterization, support interaction, and application are, herein, highlighted.

Keywords: layered chalcogenides; hydrogen evolution; $\mathrm{MoS}_{2}$; electrolysis; photolysis.

Resumen. Éste breve compendio analiza la actividad catalítica del disulfuro de molibdeno ( $\left.\mathrm{MoS}_{2}\right)$ nanoestructurado. La transición de fase del semiconductor $\mathrm{MoS}_{2}$ nanoestructurado de $2 \mathrm{H}$ (hexagonal) a 1T (trigonal) resulta en un material metaloide mediante lo que se conoce como ingeniería de defectos de superficie por exfoliación química. Se encontró que la fase 1T es activa en la reacción electrocatalítica de evolución de hidrógeno (HER por sus siglas en inglés). Varios aspectos respecto a la síntesis, caracterización, interacción con soporte, y aplicación de $\mathrm{MoS}_{2}$ son recalcados aquí.

Palabras clave: materiales laminares o de capas; evolución de hidrógeno; $\operatorname{MoS}_{2}$; electrólisis; fotólisis.
\end{abstract}

\section{Introduction}

Hydrogen $\left(\mathrm{H}_{2}\right)$, an energy-carrier, is considered as a promising candidate to replace fossil fuels due to its high energy density $\left(143 \mathrm{~kJ} \mathrm{~g}^{-1}\right)$. Its combustion, in a low temperature fuel cell, with oxygen produces water and energy. Electrochemical and photoelectrochemical water splitting are promising strategies to produce highpurity hydrogen.[1] Additionally, a substantial technological improvement is still necessary to make this process economically feasible. Most effective catalyst materials for the electrochemical water splitting are based on precious metals.[2] Moreover, the scarcity of such materials on the earth crust and their elevated cost hinder their widespread application as water splitting electrocatalysts. Research effort is being devoted to tailor novel active and stable materials made of low-cost, non-toxic, and earth-abundant catalysts. In this sense, it has been widely reported that two-dimensional (2D) layered transition metal dichalcogenides (TMD), at the nano-scale, are promising non-platinum group (NPG) catalysts for the hydrogen evolution reaction (HER). The properties of 2D materials can be extended, e.g., to photocatalysis, electrocatalysis, optics, and mechanics.[3] Moreover, tuning the chemical and physical properties of layered dichalcogenides to improve their catalytic performance represents a major stumbling block to remove. In this sense, efforts have been concerted to enhance the 
photocatalytic and the electrocatalytic properties of chalcogenide-based materials for (HER) and/or water splitting. One interesting strategy is to engineering the surface-defect by exposing, as much as possible, the edge-sites and, on the other hand, to improve the material's electrical conductivity.[4-5] This short-review attempts to summarize recent advances in layered chalcogenides employed as electrocatalysts for the hydrogen evolution reaction. The discussion is centered on the $\mathrm{MoS}_{2}$ structure and on the application of this material as electrocatalyst for the electrochemical and photochemical processes.

\section{Materials and Methods}

\section{$\mathrm{MoS}_{2}$ properties}

The chalcogenides are chemical compounds consisting in at least one chalcogen anion (X), and one electropositive element, usually a transition-metal (TM) cation. $\mathrm{MoS}_{2}$ is a layered semiconductor material $(2 \mathrm{H})$ with an indirect band-gap $\left(E_{g}\right)$ of $1.3 \mathrm{eV}$. The dichalcogenide materials consist of $2 \mathrm{D}$ or quasi-2D layers, stacked along the perpendicular direction with respect to the basal plane. Their general formula is $\mathrm{MX}_{2}$, where $\mathrm{M}=\mathrm{Mo}$, $\mathrm{Ni}, \mathrm{Co}, \mathrm{W}$, and $\mathrm{X}=\mathrm{S}$, Se or Te. A single-sheet of $\mathrm{MX}_{2}$ consists of one plane of metal-cation "sandwiched" between two layers of chalcogens, forming a tri-layer, where strong-covalent bonding characterizes the M-X interaction to form X-M-X layers. The van der Waals force acts between two adjacent X-M-X layers along the $c$-axis to form the bulk material, see Fig. 1.

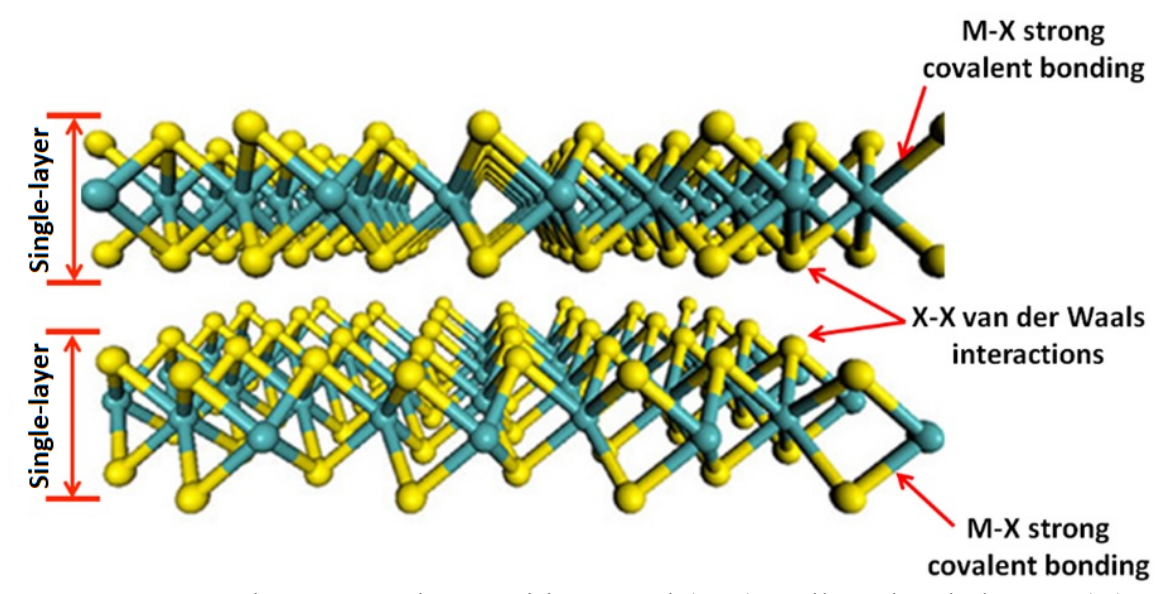

Fig. 1. The $2 \mathrm{D} M \mathrm{X}_{2}$ structure. Colors: green the transition metal (TM); yellow the chalcogen (X).

The structure of $\mathrm{MX}_{2}$ possesses two distinctive orientations: basal and edge planes with anisotropic properties. The surface inertness of the basal plane contrasts the high surface energy of the edge plane. As a consequence of this property, the electrical conductivity is ca. 2200 times higher through the layer than across the Van der Waals forces between the layers.[1] A few isolated nano-sheets of $\mathrm{MX}_{2}$ structures, at the nanoscale, present unusual chemical, mechanical, optical, and electronic properties with respect to the material in the massive state. This phenomenon is due to the electron and phonon confinement in the nano-structure.[6] In addition, the highly anisotropic properties found in the $\mathrm{MX}_{2}$ nano-structure also depend on the coordination between the transition-metal and chalcogenide, as well as on the multi-layer stacking sequence, cf. Fig. 2(a).[7] A consequence of this is that $\mathrm{MX}_{2}$ can display variable band-gap energy ranging from $0 \mathrm{eV}$ (metal-like) to 2 $\mathrm{eV}$ (semiconductor) as a result of the elemental combination, size, number of layers, and the presence or absence of dopant atoms.[8]

A single-layer $\mathrm{MX}_{2}$ may have a trigonal prismatic $(2 \mathrm{H})$ or octahedral $(1 \mathrm{~T})$ metal coordinating phase. In multiple structures, each individual layer can possess any of the two coordinating phases. Different 
polymorphic structures, with a large variety of different properties, can be represented by the same $\mathrm{MX}_{2}$. The most common polymorphs of $\mathrm{MX}_{2}$ are $1 \mathrm{~T}, 2 \mathrm{H}$ and $3 \mathrm{R}$, Fig. 2 . The number indicates the number of layers in the crystallographic unit cell, and the letter indicates the type of symmetry. T stands for tetragonal ( $\mathrm{D}_{3 \mathrm{~d}}$ group), $\mathrm{H}$ for hexagonal ( $\mathrm{D}_{3 \mathrm{~h}}$ groups), and $\mathrm{R}$ for rhombohedral $\left(\mathrm{C}_{3 \mathrm{v}}{ }^{5}\right.$ group).[9] Fig. 2 recaps the crystalline structure of $\mathrm{MX}_{2}$ and the band structure of $2 \mathrm{H}, 1 \mathrm{~T}$.
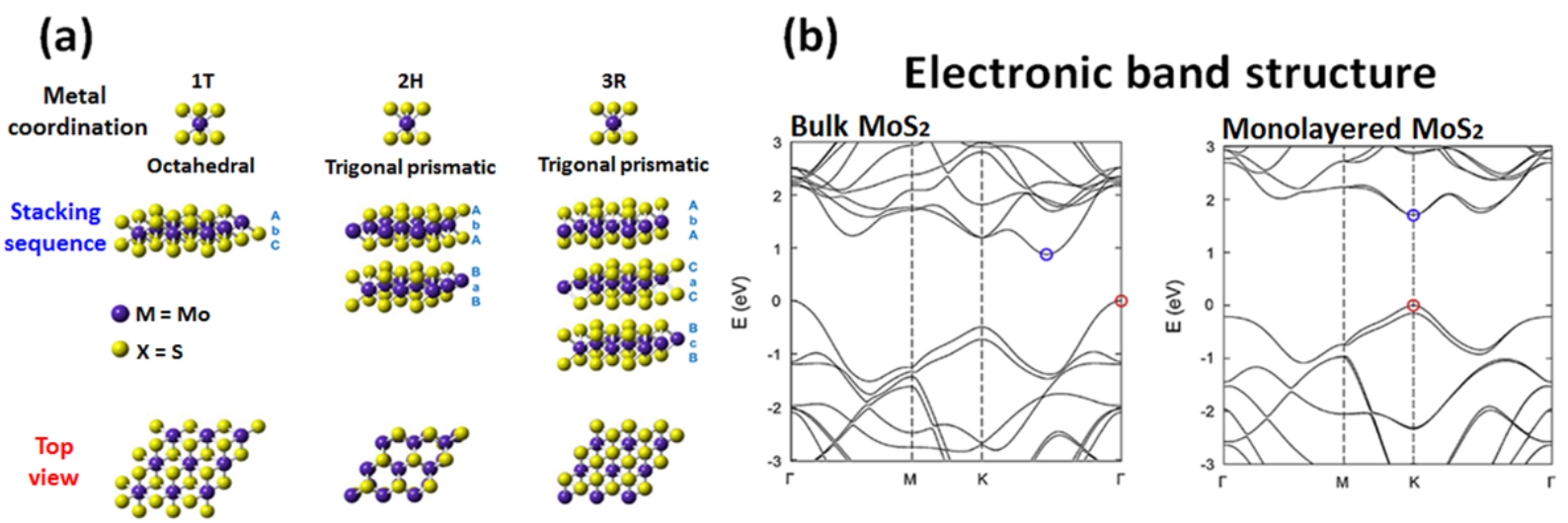

Fig. 2. (a) The metal coordination in $1 \mathrm{~T}, 2 \mathrm{H}$, and $3 \mathrm{R}$ phases of $\mathrm{MX}_{2}$ (M: Mo, W; $\left.\mathrm{X}: \mathrm{S}, \mathrm{Se}\right)$. (b) The electronic band structure of $\mathrm{MoS}_{2}$ (bulk), and $\mathrm{MoS}_{2}$ (monolayer). Figure adapted from references.[9-10]

The thermodynamically more stable $2 \mathrm{H}$ phase is most commonly found in the massive (bulk) material $\mathrm{MX}_{2}$, Fig. 2(b). The first-row of transition metals usually form the $2 \mathrm{H}$ coordinating phase, which is the most stable. The phase transition from $2 \mathrm{H}$ to $1 \mathrm{~T}$ in $\mathrm{MoS}_{2}$ is possible through the donation of electrons, which produce a change in the XPS peak of Mo $3 d$ to reduce the binding energy, by means of alkali-intercalation.[11] This phase transition is very important because the electronic properties of the materials are modified: $2 \mathrm{H}-\mathrm{MX}_{2}$ is semiconductor, whereas $1 \mathrm{~T}-\mathrm{MX}_{2}$ behaves as a metal-like material. Therefore, the structural-characterization of $\mathrm{MX}_{2}$ materials is of paramount importance to take into account for the evaluation of the electrocatalytic properties. Among all $\mathrm{MX}_{2}$ materials, molybdenum disulfide, $\mathrm{MoS}_{2}$, is the first TMD to emerge as an attractive catalyst for hydrogen evolution reaction, and the material is still explored as a prototype model to better understand the behavior of nano-structured $\mathrm{MX}_{2}$-based materials.

\section{Results and Discussion}

\section{The $\mathrm{MoS}_{2}$ properties}

In general, a single-layer of $\mathrm{MoS}_{2}$ has a laminar S-Mo-S structure with a thickness of approximately $0.7 \mathrm{~nm}$.[12] Each 2D layer of $\mathrm{MoS}_{2}$ consists of a hexagonal plane of Mo atoms and two $\mathrm{S}$ atoms of hexagonal planes modulated by the covalent interactions in trigonal prismatic form. The Mo-S length, the crystal lattice constant, and the distance between the upper and lower sulfur atoms are $2.4 \AA, 3.2 \AA$, and $3.1 \AA$, respectively. [13] The absence of the (002), (103) and (105) Bragg peaks in the X-ray diffraction pattern is characteristic of one-molecule-sheet thickness.[11-13] The two main types of $\mathrm{MoS}_{2}$ semiconductor coordination phases are 2H and $3 \mathrm{R}$. Phase $2 \mathrm{H}$ is the most thermodynamically stable. In the $1 \mathrm{~T}$ metallic phase, Mo atoms are octahedrally coordinated, possessing a higher electrical conductivity, anisotropy, chemical stability in acid environments, and high photo-corrosion resistance.[14] These properties of $\mathrm{MoS}_{2}$ are due to the anti-bonding state as a result of the interaction between Mo $d_{z^{2}}$ orbitals and S $p_{z}$ orbitals at the top of the valence band.[15] Since the bandgap energy varies with the number of layers, $[8,16]$ the band-structure is a property that can be tuned. As the thickness of $\mathrm{MoS}_{2}$ decreases to the size of a monolayer, cf. Fig. 2, a transformation from the indirect band-gap 
$(1.3 \mathrm{eV})$ to the direct band-gap $(1.9 \mathrm{eV})$ is observed.[8] Based on computational approach using the density functional theory (DFT), the suggested explanation for this band-gap transition was that the top-edge of the valence band and the bottom-edge of the conduction band, on $\operatorname{MoS}_{2}$, are located at the $\Gamma$ point, almost halfway along $\Gamma-\mathrm{K}$ direction, respectively, [10] Fig. 2(b). With the increasing number of layers, the bottom-edge of the conduction band moves upward, increasing the overall band-gap. The conduction band states at the K point are mainly associated with Mo $d$-orbitals and are relatively unaffected by the interlayer interaction. The direct bandgap at the $\mathrm{K}$ point only increases approximately $0.05 \mathrm{eV}$ to $0.1 \mathrm{eV}$. [16] The states near the $\Gamma$ point on the conduction band, due to the hybridization between S $p_{z}$-orbitals and Mo $d$-orbitals, are strongly dependent on the interlayer interaction. Hence, the bands at the $\Gamma$ point are remarkably affected by the decrease in the number of layers. Therefore, for a mono-layer structure, the indirect transition gap is larger than the direct transition gap. The direct band-gap (ca. $1.9 \mathrm{eV}$ ) at the K point becomes the smallest gap.[16]

\section{Water splitting}

The water splitting is a chemical reaction (eq. 1) in which water is separated into hydrogen and oxygen.[17] Regardless of the electrolyte, at standard conditions ( 1 bar and $298 \mathrm{~K})$, the reaction requires a minimum energy, $\Delta \mathrm{G}=237.1 \mathrm{~kJ} \mathrm{~mol}^{-1}$, or $\Delta \mathrm{E}=1.23 \mathrm{~V}$. Higher potentials (overpotential) applied to the electrodes are required to activate the intrinsic kinetic barriers present in the anode and the cathode materials. This matter is still open, since efforts are being done nowadays to tailor highly active novel materials, based on non-precious catalytic centers, to reduce the overpotential.[18]

$$
2 \mathrm{H}_{2} \mathrm{O} \rightarrow 2 \mathrm{H}_{2}+\mathrm{O}_{2}
$$

In addition to water splitting via electrolysis, the reaction can also be performed by photocatalysis.[19] The conduction band energy and valence band energy levels rely on the band-gap and the position in the electrochemical energy scale of a semiconducting material to straddle the redox potential of water, $E^{\circ} H^{+} / H_{2}$, $E^{\circ} \mathrm{O}_{2} / \mathrm{H}_{2} \mathrm{O}$, and to absorb the visible-region spectrum photons to generate efficiently $\mathrm{e}^{-} / \mathrm{h}^{+}$pairs. Moreover, an efficient cost-effective artificial photosynthesis for water splitting needs to be further developed.[20] The current status of this approach has been discussed recently.[21]

\section{Hydrogen evolution reaction electrocatalysis}

The protons (acid environment) or water molecules (alkaline environment) are reduced in a half-cell reaction to evolve to gaseous hydrogen. In acid conditions, the overall hydrogen evolution reaction (HER) is represented by eq. (2).

$$
2 H^{+}(a q)+2 e^{-} \rightarrow H_{2(g)}
$$

The activation energy of this reaction, reflected by the magnitude of the overpotential, $\eta$, is needed to overcome the energy barrier. The role of the electrocatalytic material is to decrease the activation energy, and/or the magnitude of the overpotential. In acid medium, the HER is well-established to proceed in various steps, that is:

Volmer step:

$*+H^{+}+e^{-} \rightleftharpoons * H_{a d}$

Heyrovsky step

$$
* H_{a d}+e^{-}+H^{+} \rightleftharpoons H_{2}+*
$$

Tafel step: 
where, $*$ denotes the surface-active site of the electrode.

In the classical view, the Tafel slope can suggest the reaction determining step (RDS). Tafel slopes of 120, 40 and $30 \mathrm{mV} \mathrm{dec}^{-1}$ are associated to Volmer, Heyrovsky and Tafel RDS, respectively. The HER can be either due to the Volmer-Heyrovsky, or Volmer-Tafel mechanisms. For the HER the Volmer step is the initial event involving the adsorption of hydrogen atom $\left(\mathrm{M}-\mathrm{H}_{\mathrm{ads}}\right)$ at the surface of the electrode (M). Thus, the descriptor of the reaction is the free energy of hydrogen adsorption, $\Delta G_{H}{ }^{+}$. According to the Sabatier's principle, if $\Delta G_{H}{ }^{+}$is too weak, the adsorption step will limit the overall reaction rate. Moreover, if $\Delta G_{H}{ }^{+}$is too strong, the desorption step will limit the overall reaction rate. That is why an optimal HER catalyst has values of $\Delta G_{H}{ }^{+}$ close to zero. This principle gives rise to the construction of a volcano curve, Fig. 3. This figure summarizes the HER exchange current density, $j o$, of catalytic metal centers, as function of $\Delta G_{H}{ }^{+}$.

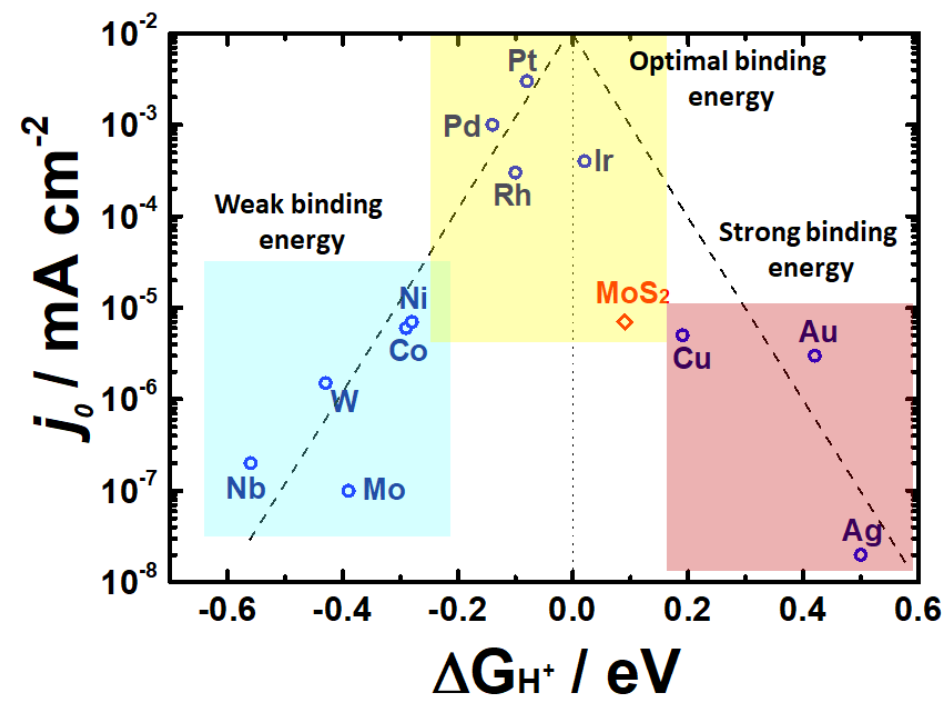

Fig. 3. The exchange current density, $j o$, as a function of the hydrogen-adsorption free energy, $\Delta G_{H^{+}}$, for selected hydrogen Evolution Reaction-metal centers. Data are displayed in a Volcano type-plot. The activity of $\mathrm{MoS}_{2}$ is also displayed. Data adapted from ref. [1].

The ideal electrocatalyst possesses properties such as high surface area, high active-sites density, high electrical conductivity, high chemical stability, optimal adsorption properties, and facile (scale-up) way of synthesis. Therefore, to optimize the HER kinetics, tailoring and tuning the properties of a catalyst is of paramount importance. In this regard, the HER mechanism on different surfaces was studied employing the DFT calculations by Hinnemann et al. [22] These authors revealed that nanostructured $\mathrm{MoS}_{2}$ possesses a $\Delta G_{H}{ }^{+}$ value close to zero, cf. Fig. 3, thus, the proton adsorption energy was comparable to that of Pt.[22] This fact indicated that $\mathrm{MoS}_{2}$ is a potential HER electrocatalyst, as demonstrated experimentally by Jaramillo et al.[23] The sulfur-edge sites of nanostructured $\mathrm{MoS}_{2}$ were identified as the real active electrocatalytic HER sites. Therefore, nanostructured $\mathrm{MoS}_{2}$, possessing a high density of sulfur-edges sites, is considered as a highly active material for the HER. Moreover, the design and fabrication of $\mathrm{MoS}_{2}$-based materials, with a significant activity and stability towards the HER still remain a challenge. Consequently, several strategies such as the use of nanoparticles and nano-sheets by defect-surface engineering,[24] $\operatorname{MoS}_{\mathrm{x}}$ amorphous-like films ${ }^{14}$ the 
hybridization with some transition-metal, e.g., $\mathrm{Ni}[25]$ or $\mathrm{Co}[26]$ have been considered. Moreover, a very useful strategy (phase transition $2 \mathrm{H}$ to $1 \mathrm{~T}$ ), which consists in intercalating $\mathrm{Li}$-ions in $\mathrm{MoS}_{2}$ to obtain $1 \mathrm{~T} \mathrm{MoS} 2$ nanoparticles and/or nano-sheets, has been widely used.[24-27] For example, Lukowski et al.[27] prepared 1T-MoS 2 nano-sheets grown onto graphite via a chemical vapor deposition method. They observed that the HER kinetics dramatically enhanced, reporting a Tafel slope of $43 \mathrm{mV} \mathrm{dec}^{-1}$, and a cathodic overpotential of $187 \mathrm{mV}$ at 10 $\mathrm{mA} \mathrm{cm}{ }^{-2}$. The authors attributed the HER enhancement to the metallic-like behavior, and a high density of defects generated on 1T-MoS 2 structure.[27] In spite of this phenomenon, 1T-MoS 2 nano-sheets showed a poor stability,[14] due to the reconversion of $1 \mathrm{~T}$ to $2 \mathrm{H}$ semiconducting phase, a phenomenon that occurs even at moderated-annealing-temperature of $95^{\circ} \mathrm{C}$.

On the other hand, the electrochemical impedance spectroscopy (EIS) analysis of MoSx films, electrodeposited onto glassy carbon disks, suggested an electronic coupling between the amorphous molybdenum sulphide and the conductive carbon material, as claimed by Vrubel et al.[28] Such coupling was important to enhance the kinetics towards HER. Since the low conductivity of $\mathrm{MoS}_{2}$ is a problem, ${ }^{1}$ chemicallyexfoliated $\mathrm{MoS}_{2}$ nano-sheets with a high concentration of metallic 1T phase and the presence of semiconducting $2 \mathrm{H}$ phase was reported by Voiry et al.[29] Both phases were integrated with carbon nanotubes and were evaluated towards the HER in $0.5 \mathrm{M} \mathrm{H}_{2} \mathrm{SO}_{4}$. These structures showed a high performance towards HER, with a Tafel slope of $40 \mathrm{mV} \mathrm{dec}{ }^{-1}$, assessing the importance of the electrical conductivity enhancement. Therefore, the integration of $\mathrm{MoS}_{2}$-nanostructures with conductive support-materials is an effective way to activate the catalytic centers. These nanostructures justify the fact of mediating the growth, dispersing catalytic nanoparticles, and providing electrons for the electrocatalytic activation. Taking advantage of the properties of reduced graphene oxide, RGO, Li et al.,[30] prepared hybrid materials: $\mathrm{MoS}_{2} / \mathrm{RGO}$. Their results revealed that the integration of RGO with $\mathrm{MoS}_{2}$ also enhanced significantly the HER kinetics in acid, due to a rich active edge site of the small particle size, and the "synergy effect", i.e., the interaction effect arising between $\mathrm{MoS}_{2}$ and RGO (acting as support). However, the "synergy effect" between $\mathrm{MoS}_{2}$ and carbon support-materials was not further discussed. Although the limited electrical conductivity of $\mathrm{MoS}_{2}$ has been circumvented by coupling the TMD with carbon support materials, there are not enough studies discussing the interaction of the catalytic center with the support. Some hints are, moreover, provided by Niu et al.[31] The synchrotron X-Ray Absorption Spectroscopy (XAS) was used to study the synergy effect between nanoplates of $\mathrm{MoS}_{2}$, with a high density of unsaturated sulphur-edges and a short distance between basal planes to edges, on carbon nanofiber, $\mathrm{CNF}$, see inset in Fig. 4(a). The CNF in the system $\mathrm{MoS}_{2} / \mathrm{CNF}$ receives more electrons in the high-energy $\sigma^{*}$ states than in the low-energy $\pi^{*}$ states, resulting in a more weighted unoccupied $\pi^{*}$ states. In addition, as further

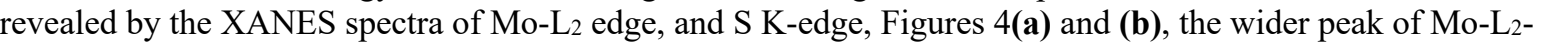
edge suggests electron transfer of Mo $4 d$ to $\mathrm{C} 2 p$ states, as a result of the interaction of the TMD with the support, whereas on the S K-edge, besides the signal of the TMD (peak A) the enhanced peak B put in evidence the reactivity of $\mathrm{S}$ at the edges with oxygen. Summing up, the interaction phenomenon increases the graphitic character of $\mathrm{CNF}$ in the $\mathrm{MoS}_{2} / \mathrm{CNF}$ system, favoring the conduction of electrons and thus the HER kinetics, Fig. 4(d). On the other hand, photocatalysis is also favored on the same system due to the electronic interaction between $\mathrm{MoS}_{2}$ and CNF via the presence of a photosensitizer (FL: fluorescein), Fig. 4(c). These results contrast that, by inducing a strong $\mathrm{MoS}_{2}$-carbon interaction, the overall HER performance is increased. 

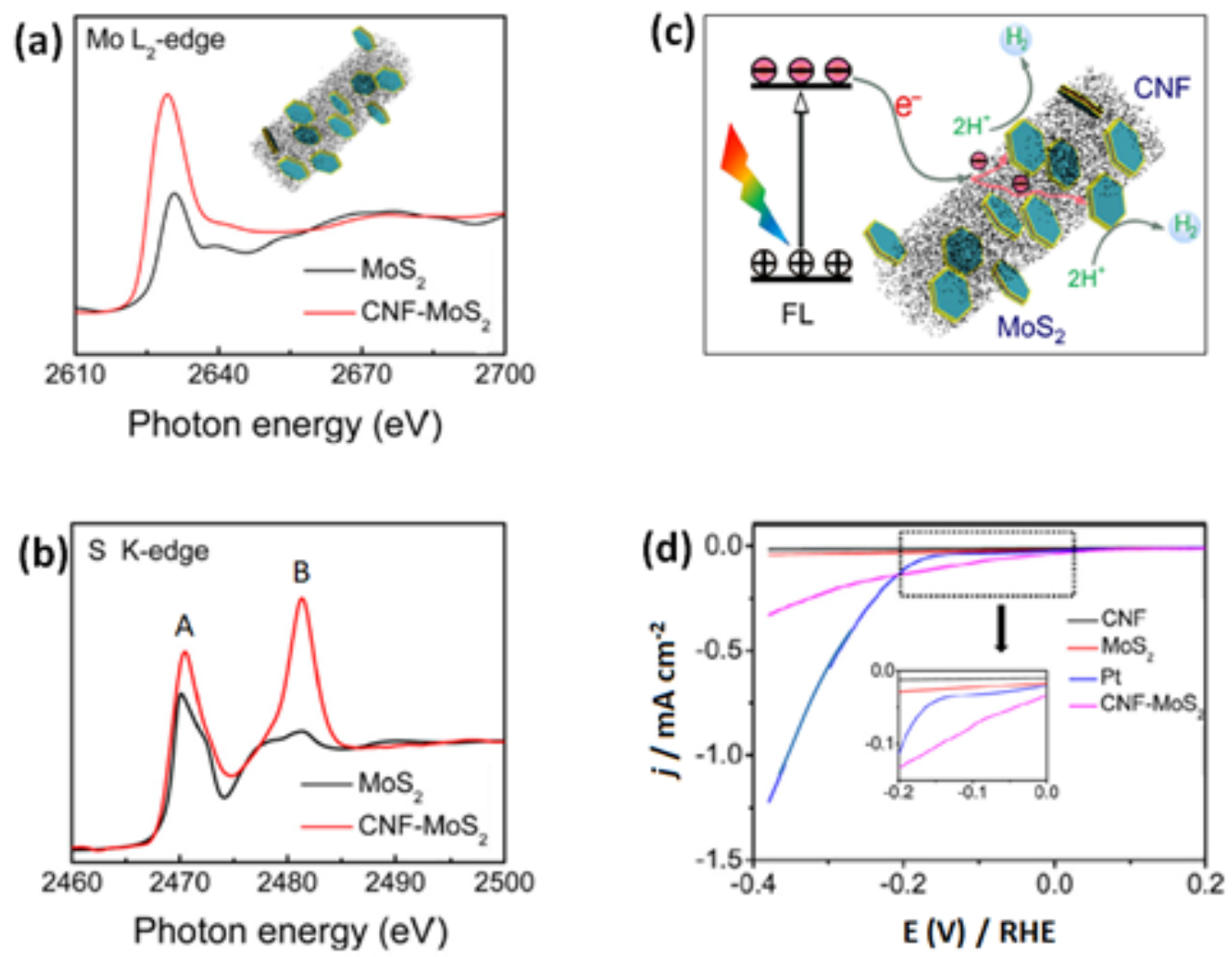

Fig. 4. For the system $\mathrm{MoS}_{2}-\mathrm{CNF}$ : (a) Mo-L $\mathrm{L}_{2}$ edge XANES spectra. (b) S-K edge XANES spectra. (c) photoelectrocatalytic HER on $\mathrm{MoS}_{2}-\mathrm{CNF}$, photosensitized by the presence of fluorescein (FL). (d) The electrocatalytic HER on CNF, $\mathrm{MoS}_{2}, \mathrm{Pt}$ and $\mathrm{MoS}_{2}-\mathrm{CNF}$ materials. Data adapted from ref. [31]

In order to compare the performance of $\mathrm{MoS}_{2}$-based catalysts, the Tafel slope and the overpotential to drive $-10 \mathrm{~mA} \mathrm{~cm}^{-2}, \eta_{@ 10}$, are considered. The $\eta_{@ 10}$ is the electrode-activity parameter considered as an optimal metric for solar fuel applications.[1] The Tafel slope determines the additional potential required to increase the kinetic current by an order of magnitude. Thus, low Tafel slope values are desirable (as close as possible to $30 \mathrm{mV} \mathrm{dec}{ }^{-1}$ ), specifically for high-current applications, e.g., in electrolyzer devices. Fig. 5 summarizes HER activity state-of-the-art collected data so far on $\mathrm{MoS}_{2}$ electrocatalysts in acid medium. We can clearly appreciate the positive evolution of $\mathrm{MoS}_{2}$-based catalysts based on the materials' engineering between the catalytic center and the support. This approach influences the HER mechanism. As discussed in the literature, the defect engineering and change of the phase transition to $1 \mathrm{~T}$ coordination assure a robust surface defect-density, identified as the real active sites for HER. Unfortunately, the stability of such nano-structures, as well as their electrical conductivity are still to be improved. This is the reason, to this day, that the coupling with conductive carbon supports of $\mathrm{MoS}_{2}$-structures offer the highest HER performance. To our best knowledge, the strong interaction between the catalytic center and the support can improve the activity and stability of the catalytic center. An approach that has to be considered in the majority of nanoparticulated electrocatalytic centers to enhance reactions of technological importance. 


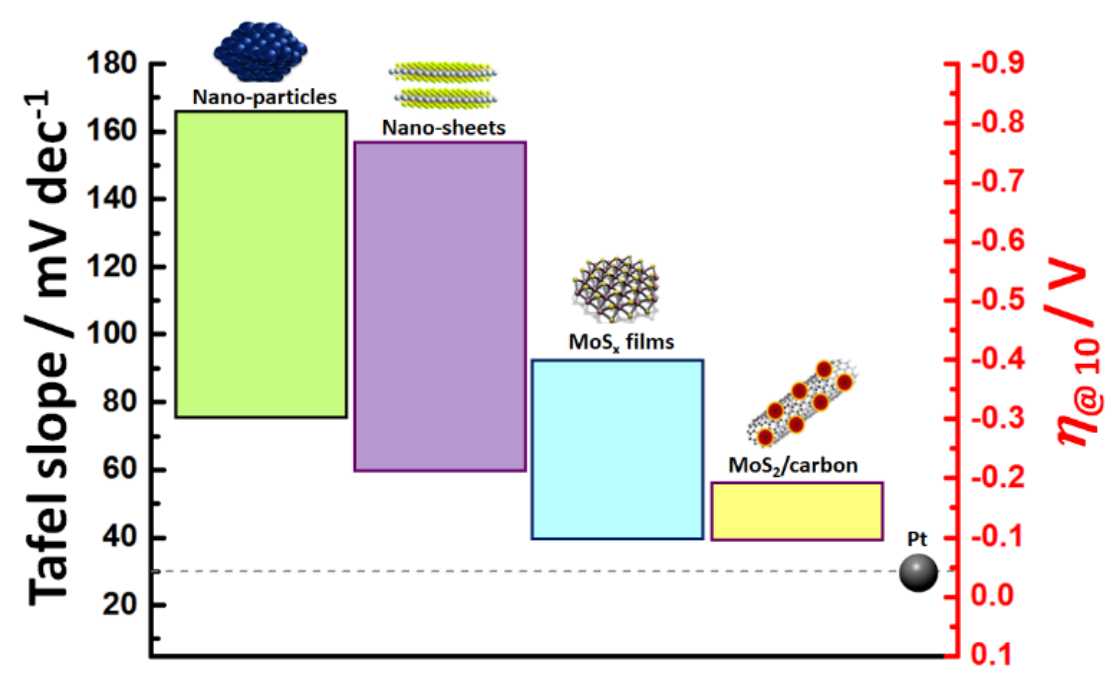

Fig. 5. HER on $\mathrm{MoS}_{2}$-based catalysts state-of-the-art in acid medium. The height of each box highlights the scattered Tafel slope data reported by various laboratories collected from the list of references.

\section{Photocatalysis of the hydrogen evolution reaction}

A reference material which is widely used for the photoelectrocatalytic hydrogen production is the $\mathrm{TiO}_{2}$ (anatase phase) decorated with a metal co-catalyst, e.g., Pt. The performance determined by the optical limit under AM1.5 Global irradiation of oxides (e.g., $\mathrm{TiO}_{2}, \mathrm{Fe}_{2} \mathrm{O}_{3}, \mathrm{WO}_{3}$ ) is extremely low for the overall water splitting.[32] Moreover, a constant effort has demonstrated that chalcogenides ( $\mathrm{MoS}_{2}$-based) are attractive materials for water splitting photoelectrocatalysis. The p-type conductivity of $\mathrm{MoS}_{2}$ films $\left(\mathrm{E}_{\mathrm{g}}=1.87 \mathrm{eV}\right)$ has been reported by Liu et al.[33] Therefore, p-n heterojunctions based p-MoS 2 and $n-C d S\left(E_{g}=2.4 \mathrm{eV}\right)$, Fig. 6(a), were efficient and stable for the HER under visible irradiation.[16] The photogenerated electrons $\left(\mathrm{e}^{-}\right)$at the conduction band (CB) of $\mathrm{MoS}_{2}$ are conveyed to the counter electrode (where HER proceeds) via the CdS CB, whereas the photogenerated holes $\left(\mathrm{h}^{+}\right)$at the $\mathrm{CdS}$ valence band (VB) are transferred to the $\mathrm{MoS}_{2} \mathrm{VB}$ avoiding the photo-corrosion of CdS.
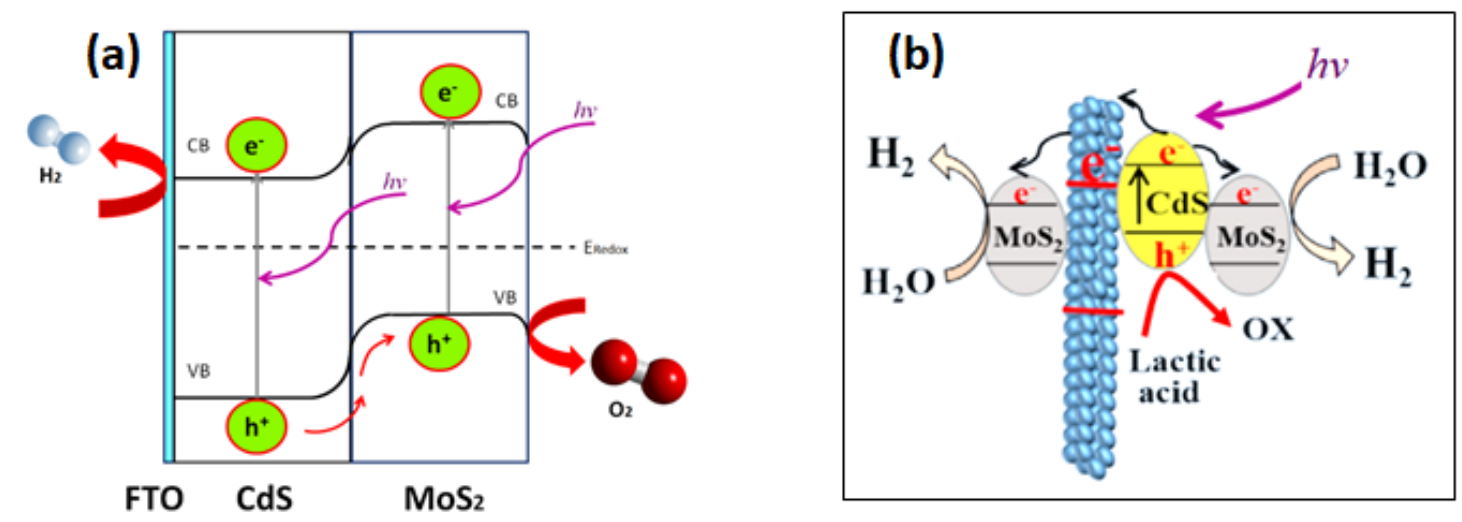

Fig. 6. (a) p-n $\mathrm{MoS}_{2} / \mathrm{CdS}$ heterojunction for the water splitting under visible irradiation. Adapted from ref. [33]; (b) Photocatalytic hydrogen evolution under visible light, and a sacrificing agent - hole scavenger (Lactic acid). Adapted from ref. [35] 
A similar heterojunction produced by electrospinning mediated photo-deposition technique using oxide nanofibers $\left(\mathrm{TiO}_{2}-\mathrm{NF}\right)$ was developed by Qin et al.[34] The resulting system schematized in Fig. 6(b) is $\mathrm{MoS}_{2} / \mathrm{CdS}-\mathrm{TiO}_{2}-\mathrm{NF}$. Alike the system shown in Fig. 6(a), the $\mathrm{MoS}_{2} / \mathrm{CdS}-\mathrm{TiO}_{2}-\mathrm{NF}$ system was designed for the visible-light photocatalytic hydrogen production. A sacrificing agent (lactic acid) serving as hole scavenger was used. Under this condition, the $\mathrm{H}_{2}$ evolution was 3 - 4 times higher on $\mathrm{MoS}_{2} / \mathrm{CdS}-\mathrm{TiO}_{2}-\mathrm{NF}$ than on $\mathrm{MoS}_{2} / \mathrm{CdS}-$ $\mathrm{P} 25$ and $\mathrm{Pt} / \mathrm{CdS}-\mathrm{TiO}_{2}$.

Taking advantage of the unique electrical properties of 1D and 2D carbon nano-structures, [35] nanostructures based on the TMD and carbon, photocatalysts were designed for the photocatalytic hydrogen evolution. As shown in Fig. 4, nanohybrids by nanoplates deposition of $\mathrm{MoS}_{2}$ nano-structures onto carbon nanofibers and a photosensitizer (FL) were evaluated toward the photocatalytic hydrogen production by Niu et al.[31] The HER rate increased considerably as a result of the synergy effects of the semiconductor with the carbon support. Moreover, a high HER photo-activity was observed on $\mathrm{MoS}_{2} /$ graphene as co-catalyst on $\mathrm{TiO}_{2}$ nano-crystals.[15] The presence of graphene network in this system facilitated the charge separation generated at the semiconducting materials thus avoiding, the charge recombination, and increasing the hydrogen production rate by a factor of 39, i.e., 4 times higher than the rate on $\mathrm{TiO}_{2}$, and on $\mathrm{TiO}_{2}-\mathrm{MoS}_{2}$. The consensus on carbon (graphene) on $\mathrm{MoS}_{2}$-carbon, as HER photo-catalysts, as activity enhancer is due to the extended charge separation, less charge recombination on this graphene network, as assessed on various reports.[2,16,35]. This is certainly an avenue, and the reason why future works should address the interaction between the catalytic center, e.g., $\mathrm{MoS}_{2}$ and the support.

\section{Conclusion}

Layered-based nanostructured TMD are promising materials for electrocatalysis of HER and photocatalysis for water splitting technology. Understanding the properties of such nano-structures is extremely important to tailor and tune their performance towards electrocatalytic processes. A widely studied example is the limited electrical conductivity of molybdenum disulfide. Although many authors claim that the coupling between carbon surfaces and $\mathrm{MoS}_{2}$ catalytic-centers enhances the HER kinetics, there are very few studies which discuss deeply this important "ingredient" to tune a high active and stable HER catalyst. To the best of our knowledge, the strong interaction between the catalytic center and its support can improve the activity and stability of a given photoelectrocatalytic material. Therefore, inducing a strong $\mathrm{MoS}_{2}$-carbon interaction is proposed to be a novel strategy in the $\mathrm{MoS}_{2}$ performance towards HER.

\section{Acknowledgment}

The authors acknowledge the support of the University of Poitiers. CA.C.R. acknowledges financial support from CONACyT-Mexico Scholarship Nr. MX-561206.

\section{References}

1. Benck, J. D.; Hellstern, T. R.; Kibsgaard, J.; Chakthranont, P.; Jaramillo, T. F. ACS Catalysis 2014, 4 (11), 3957-3971.

2. Li, X.; Hao, X.; Abudula, A.; Guan, G. Journal of Materials Chemistry A 2016, 4 (31), 11973-12000.

3. Manzeli, S.; Ovchinnikov, D.; Pasquier, D.; Yazyev, O. V.; Kis, A. Nature Reviews Materials 2017, $2(8), 17033$.

4. Yang, J.; Shin, H. S. J. Mater. Chem. A 2014, 2 (17), 5979-5985. 
5. (a) Peng, K.; Fu, L.; Yang, H.; Ouyang, J.; Tang, A. Nano Research 2016, 10 (2), 570-583; (b) Lee, Y. H.; Zhang, X. Q.; Zhang, W.; Chang, M. T.; Lin, C. T.; Chang, K. D.; Yu, Y. C.; Wang, J. T.; Chang, C. S.; Li, L. J.; Lin, T. W. Advanced materials 2012, 24 (17), 2320-5.

6. Deng, D.; Novoselov, K. S.; Fu, Q.; Zheng, N.; Tian, Z.; Bao, X. Nature nanotechnology 2016, 11 (3), 218-30.

7. Anantharaj, S.; Ede, S. R.; Sakthikumar, K.; Karthick, K.; Mishra, S.; Kundu, S. ACS Catalysis 2016, $6(12), 8069-8097$.

8. Lee, H. S.; Min, S. W.; Chang, Y. G.; Park, M. K.; Nam, T.; Kim, H.; Kim, J. H.; Ryu, S.; Im, S. Nano letters 2012, 12 (7), 3695-700.

9. Chia, X.; Eng, A. Y.; Ambrosi, A.; Tan, S. M.; Pumera, M. Chemical reviews 2015, 115 (21), 11941 66.

10. Yazyev, O. V.; Kis, A. Materials Today 2015, 18 (1), 20-30.

11. Wang, X.; Shen, X.; Wang, Z.; Yu, R.; Chen, L. ACS Nano 2014, 8 (11), 11394-1140.

12. Late, D. J.; Liu, B.; Matte, H. S. S. R.; Dravid, V. P.; Rao, C. N. R. ACS Nano 2012, 6 (6), 5635-5641.

13. Ataca, C.; Şahin, H.; Ciraci, S. The Journal of Physical Chemistry C 2012, 116 (16), 8983-8999.

14. Yan, Y.; Xia, B.; Xu, Z.; Wang, X. ACS Catalysis 2014, 4 (6), 1693-1705.

15. Xiang, Q.; Yu, J.; Jaroniec, M. Journal of the American Chemical Society 2012, 134 (15), 6575-8.

16. Han, B.; Hu, Y. H. Energy Science \& Engineering 2016, 4 (5), 285-304.

17. (a) Trasatti, S. Int J Hydrogen Energy 1995, 20 (10), 835-844; (b) de Levie, R. Journal of Electroanalytical Chemistry 1999, 476, 92 - 93.

18. (a) Kanan, M. W.; Surendranath, Y.; Nocera, D. G. Chemical Society reviews 2009, 38 (1), 109-14;

(b) Zhong, H.; Tian, R.; Gong, X.; Li, D.; Tang, P.; Alonso-Vante, N.; Feng, Y. Journal of Power Sources 2017, 361, 21-30; (c) Chaudhari, N. K.; Jin, H.; Kim, B.; Lee, K. Nanoscale 2017, 9 (34), 12231-12247.

19. (a) Fujishima, A.; Honda, K. Nature 1972, 238 (5358), 37-38; (b) Nozik, A. J. Nature 1975, 257 (5525), 383-386.

20. (a) Blankenship, R. E.; Tiede, D. M.; Barber, J.; Brudvig, G. W.; Fleming, G.; Ghirardi, M.; Gunner, M. R.; Junge, W.; Kramer, D. M.; Melis, A.; Moore, T. A.; Moser, C. C.; Nocera, D. G.; Nozik, A. J.; Ort, D. R.; Parson, W. W.; Prince, R. C.; Sayre, R. T. Science 2011, 332 (6031), 805-9; (b) Nocera, D. Acc Chem Res 2012, 45 (5), 767-776; (c) Faunce, T.; Styring, S.; Wasielewski, M. R.; Brudvig, G. W.; Rutherford, A. W.; Messinger, J.; Lee, A. F.; Hill, C. L.; deGroot, H.; Fontecave, M.; MacFarlane, D. R.; Hankamer, B.; Nocera, D. G.; Tiede, D. M.; Dau, H.; Hillier, W.; Wang, L.; Amal, R. Energy \& Environmental Science 2013, 6 (4), 1074.

21. Sheng, C.; Wei, L.; Yanfa, Y.; Thomas, H.; Ishiang, S.; Dunwei, W.; Zetian, M. Nano Futures 2017, $1(2), 022001$.

22. Hinnemann, B.; Moses, P.; Bonde, J.; Jørgensen, K.; Nielsen, J.; Horch, S.; Chorkendorff, I.; Nørskov, J. Journal of the American Chemical Society 2005, 127, 5308-5309.

23. Jaramillo, T.; Jørgensen, K.; Bonde, J.; Nielsen, J.; Horch, S.; Chorkendorff, I. Science 2007, 137, 100-102.

24. (a) Ye, G.; Gong, Y.; Lin, J.; Li, B.; He, Y.; Pantelides, S. T.; Zhou, W.; Vajtai, R.; Ajayan, P. M. Nano letters 2016, 16 (2), 1097-103; (b) Lin, L.; Miao, N.; Wen, Y.; Zhang, S.; Ghosez, P.; Sun, Z.; Allwood, D. A. ACS Nano 2016, 10 (9), 8929-37; (c) Wu, Z.; Fang, B.; Wang, Z.; Wang, C.; Liu, Z.; Liu, F.; Wang, W.; Alfantazi, A.; Wang, D.; Wilkinson, D. P. ACS Catalysis 2013, 3 (9), 2101-2107; (d) Guo, B.; Yu, K.; Li, H.; Song, H.; Zhang, Y.; Lei, X.; Fu, H.; Tan, Y.; Zhu, Z. ACS applied materials \& interfaces 2016, 8 (8), 5517-25.

25. Escalera-Lopez, D.; Niu, Y.; Yin, J.; Cooke, K.; Rees, N. V.; Palmer, R. E. ACS Catalysis 2016, 6 (9), 6008-6017.

26. (a) Dai, X.; Du, K.; Li, Z.; Liu, M.; Ma, Y.; Sun, H.; Zhang, X.; Yang, Y. ACS applied materials \& interfaces 2015, 7 (49), 27242-53; (b) Staszak-Jirkovsky, J.; Malliakas, C. D.; Lopes, P. P.; Danilovic, N.; Kota, S. S.; Chang, K. C.; Genorio, B.; Strmenik, D.; Stamenkovic, V. R.; Kanatzidis, M. G.; Markovic, N. M. Nature materials 2016, 15 (2), 197-203.

27. Lukowski, M. A.; Daniel, A. S.; Meng, F.; Forticaux, A.; Li, L.; Jin, S. Journal of the American Chemical Society 2013, 135, 10274. 
28. Vrubel, H.; Moehl, T.; Gratzel, M.; Hu, X. Chem Commun 2013, 49 (79), 8985-7.

29. Voiry, D.; Salehi, M.; Silva, R.; Fujita, T.; Chen, M.; Asefa, T.; Shenoy, V. B.; Eda, G.; Chhowalla, M. Nano letters 2013, 13 (12), 6222-7.

30. Li, Y.; Wang, H.; Xie, L.; Liang, Y.; Hong, G.; Dai, H. Journal of the American Chemical Society 2011, 133 (19), 7296-9.

31. Niu, F.; Dong, C.-L.; Zhu, C.; Huang, Y.-C.; Wang, M.; Maier, J.; Yu, Y.; Shen, S. Journal of Catalysis 2017, 352, 35-41.

32. Chen, Z.; Jaramillo, T. F.; Deutsch, T. G.; Kleiman-Shwarsctein, A.; Forman, A. J.; Gaillard, N.; Garland, R.; Takanabe, K.; Heske, C.; Sunkara, M.; McFarland, E. W.; Domen, K.; Miller, E. L.; Turner, J. A.; Dinh, H. N. J. Mater. Res. 2010, 25 (1), 3-16.

33. Liu, Y.; Yu, Y.-X.; Zhang, W.-D. The Journal of Physical Chemistry C 2013, 117 (25), 12949-12957.

34. Qin, N.; Xiong, J.; Liang, R.; Liu, Y.; Zhang, S.; Li, Y.; Li, Z.; Wu, L. Applied Catalysis B: Environmental 2017, 202, 374-380.

35. Xiang, Q.; Cheng, B.; Yu, J. Angewandte Chemie 2015, 54 (39), 11350-66. 